\title{
A Colo-Urachal-Scrotal Fistula in a 55-Year-0ld Male
}

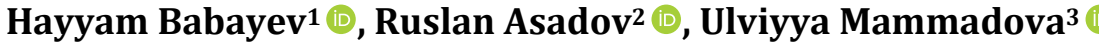 \\ ${ }^{1}$ Baku Medical Plaza Clinic, Department of General Surgery, Baku, Azerbaijan \\ ${ }^{2}$ Baku Medical Plaza Clinic, Department of Radiology, Baku, Azerbaijan \\ ${ }^{3}$ Baku Medical Plaza Clinic, Department of Urology, Baku, Azerbaijan \\ Email: hayyambabayev@gmail.com,dr.esedov@gmail.com, ronnyi@mail.ru
}

How to cite this paper: Babayev, H., Asadov, R. and Mammadova, U. (2021) A Colo-Urachal-Scrotal Fistula in a 55-Year-Old Male. Open Journal of Gastroenterology, 11, 67-73.

https://doi.org/10.4236/ojgas.2021.114007

Received: March 17, 2021

Accepted: April 27, 2021

Published: April 30, 2021

Copyright $\odot 2021$ by author(s) and Scientific Research Publishing Inc. This work is licensed under the Creative Commons Attribution International License (CC BY 4.0).

http://creativecommons.org/licenses/by/4.0/

\begin{abstract}
Introduction: The urachus or median umbilical ligament is a fibrous cord originating from the allantoic canal's involution. It extends from the bladder dome to the posterior umbilicus. A partial or a total defect of the urachus channel's obliteration after the fifth month of gestation can be the origin of urachal abnormalities. A complication of symptomatic urachal anomalies occurs when the cyst fistulizes to adjacent viscera. We report the first case of a diverticulosis-related sigmoid-urachal-scrotal cyst in a 55-year-old patient. Case Report: A 55-year-old male visited the clinic with intermittent fever, lower abdominal pain, swelling in the groin and scrotal area. Abdominal examination revealed a diffuse tender in the lower abdomen and suprapubic area. Non-contrast abdominal CT imaging showed a cystic lesion with an air-fluid level and possibly debris positioned superiorly to the bladder at the abdominal wall. The sigmoid colon seemed attached to this cystic lesion. Air observed in the scrotum and subcutaneous. The damaged part of the sigmoid colon was repaired. Then the abscess was drained. Urachus was wholly resected and removed. After five days of follow-up, the patient was discharged without any problem. A severely inflamed urachus was found in pathology. Conclusion: Although it is rare and its symptoms are non-specific, the urachal abscess should be suspected if there is persistent fever and suprapubic pain, especially in patients with diverticula. It is recommended to remove it when the diagnosis is made, considering the complications that may occur.
\end{abstract}

\section{Keywords}

Urachus, Fistula, Sigmoid Colon, Scrotum

\section{Introduction}

The urachus or median umbilical ligament is a fibrous cord that originates from 
the involution of the allantoic canal. It extends from the bladder dome to the posterior umbilicus. A partial or total defect of the urachus channel's obliteration after the fifth month of gestation can be the origin of urachal abnormalities. In some literature, the alternating sinus, which can drain both the umbilicus and bladder, has been described. Urachal problems are much less common in older children or adults. These include lower belly pain, fever, pain on urination, a urinary tract infection, or blood in the urine. A complication of symptomatic urachal anomalies occurs when the cyst fistulizes to adjacent viscera. Few enteric-urachal fistulas have been described in the literature [1]. Colo-urachal-skin fistula is also found [2].

Diverticulitis refers to inflammation and infection associated with a diverticulum. It is estimated to occur in $10 \%$ to $25 \%$ of people with diverticulosis. Peridiverticular and pericolic infection results from a perforation (either macroscopic or microscopic) of a diverticulum, which leads to contamination, inflammation, and infection. Complicated diverticulitis includes diverticulitis with abscess, obstruction, diffuse peritonitis (free perforation), or fistulas between the colon and adjacent structures. Colovesical, colovaginal, and coloenteric fistulas are long-term sequelae of complicated diverticulitis [3].

We report the first case of a diverticulosis-related sigmoid-urachal-scrotal cyst in a 55 -year-old patient.

\section{Presentation of Case}

A 55-year-old male visited the outpatient clinic with intermittent fever, lower abdominal pain, and vomiting. The patient also had swelling in the groin and scrotal area. The patient reported no change in bowel habits and reported no dysuria, changes in frequency, hematuria, and decreased flow. He was not subjected to another complaint. On physical examination, he looked tired, and his body temperature was $38.5^{\circ} \mathrm{C}$. Biochemical analysis revealed that the white blood cell (WBC) count was 18,000/mL (4000 - 8000), and the other parameters were in a normal range. Abdominal examination revealed a diffuse tender in the lower abdomen and suprapubic area.

Non-contrast abdominal computed tomography imaging showed a cystic lesion with an air-fluid level and possibly debris positioned superiorly to the bladder at the abdominal wall (Figure 1). The sigmoid colon seemed attached to this cystic lesion. Air was observed in the scrotum and subcutaneous (Figure 2).

\section{Surgery and Pathological Examination}

Preoperatively, cephalosporin (intravenous ceftriaxone 1 gr) and intravenous fluid therapy were administered. Examination of the abdominal cavity was revealed that the urachus attached to the abdominal wall and had an abscess inside. At first, the sigmoid colon was separated from the urachus. The damaged part of the sigmoid colon was repaired (Figure 3). Then the abscess was drained. The urachus was completely resected and removed (Figure 4). The abscess in 


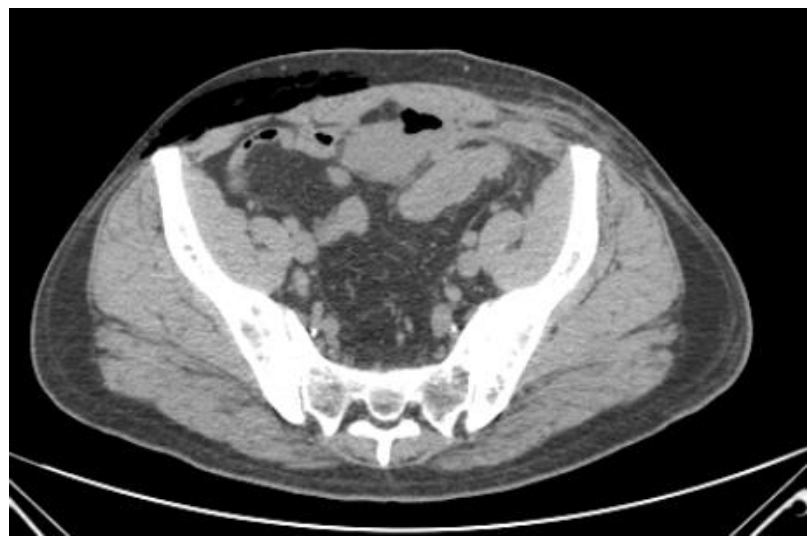

Figure 1. Air under the skin and urachus. Abdominal CT (transverse section).

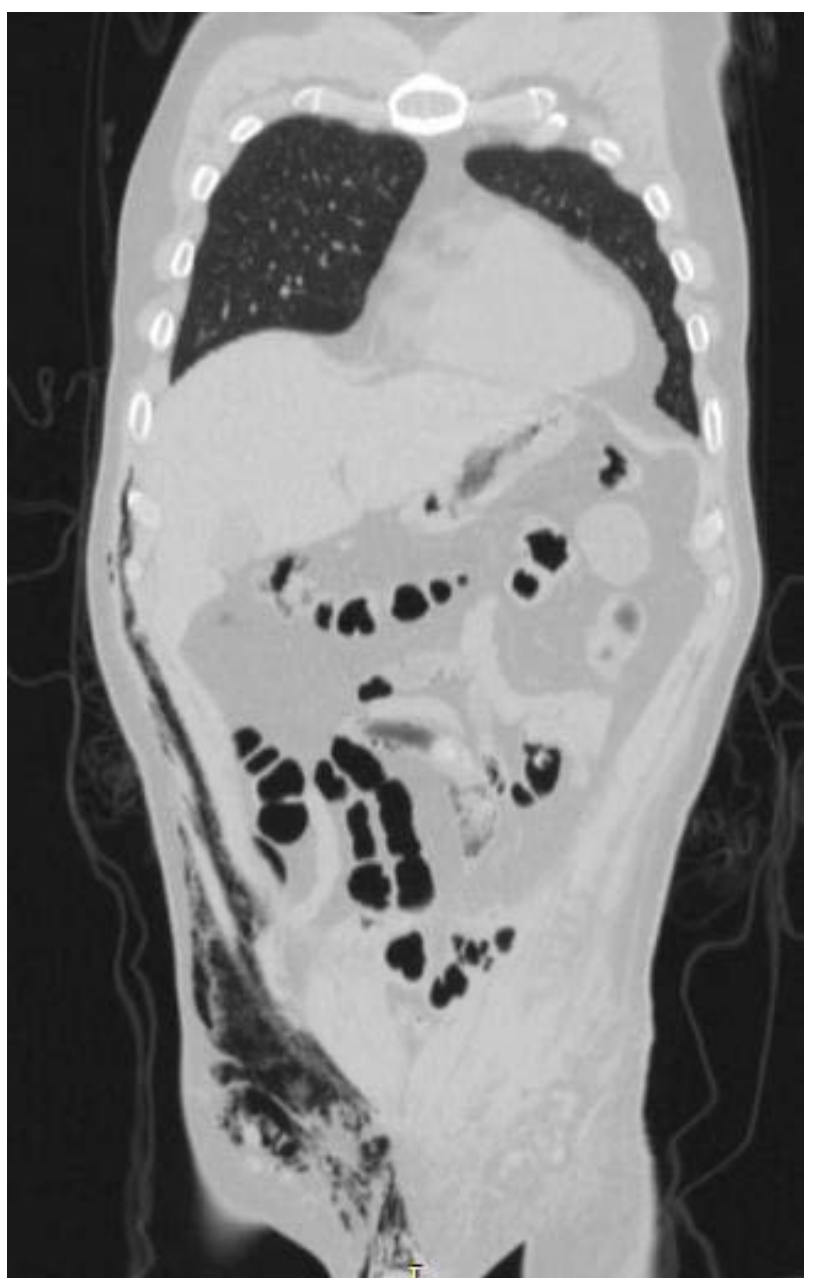

Figure 2. Air under the skin. Abdominal CT (coronal section).

the inguinal canal and scrotum was drained by the urologist (Figure 5). The surgery was completed by placing a drain in the abdomen. No complication was observed in the postoperative period. Oral intake was resumed on postoperative day 2. And the drain was removed on postoperative day 3. On postoperative day 


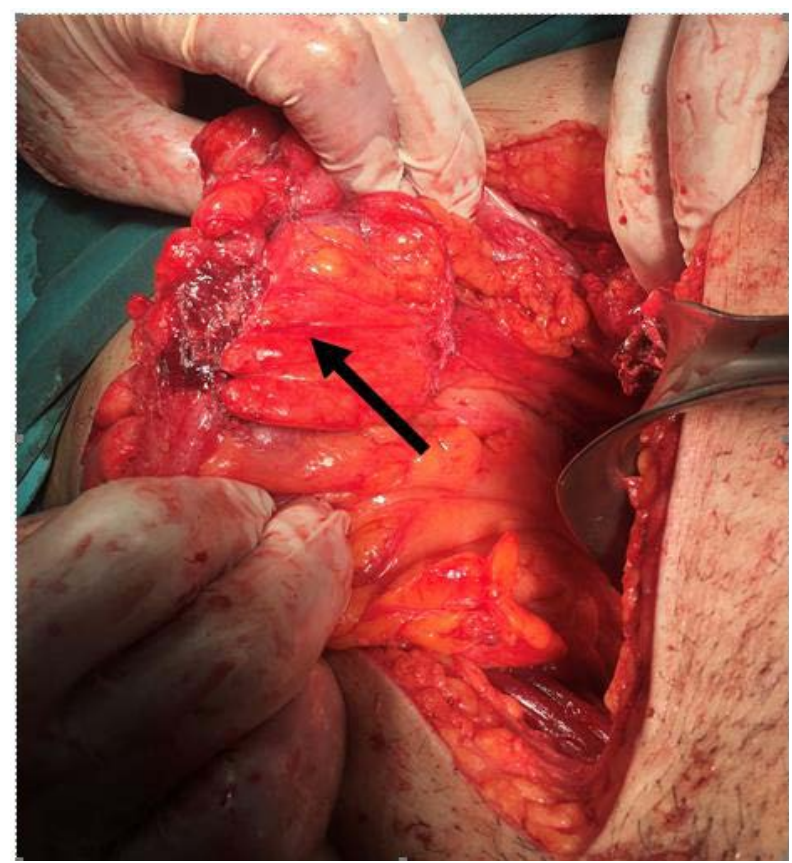

Figure 3. Edematous sigmoid colon separated from uracus.

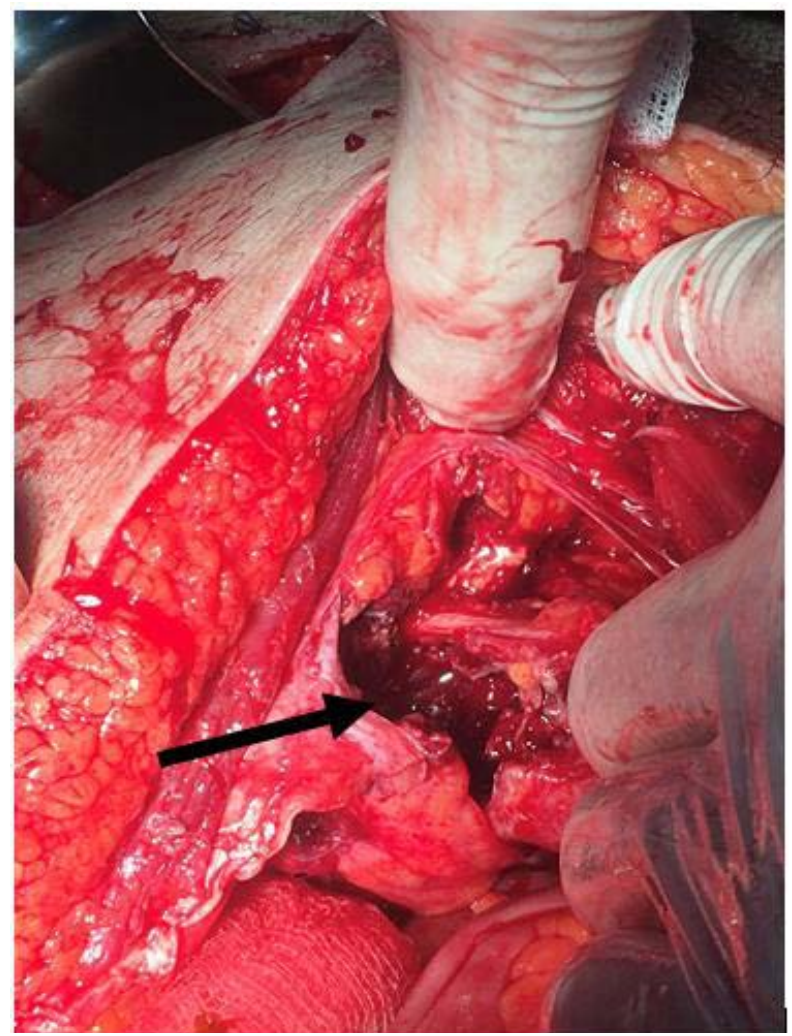

Figure 4. Drained uracus cavity.

5 , the patient recovered without complication. The symptoms were improved, the laboratory parameters were normal, and the patient was uneventfully discharged. 


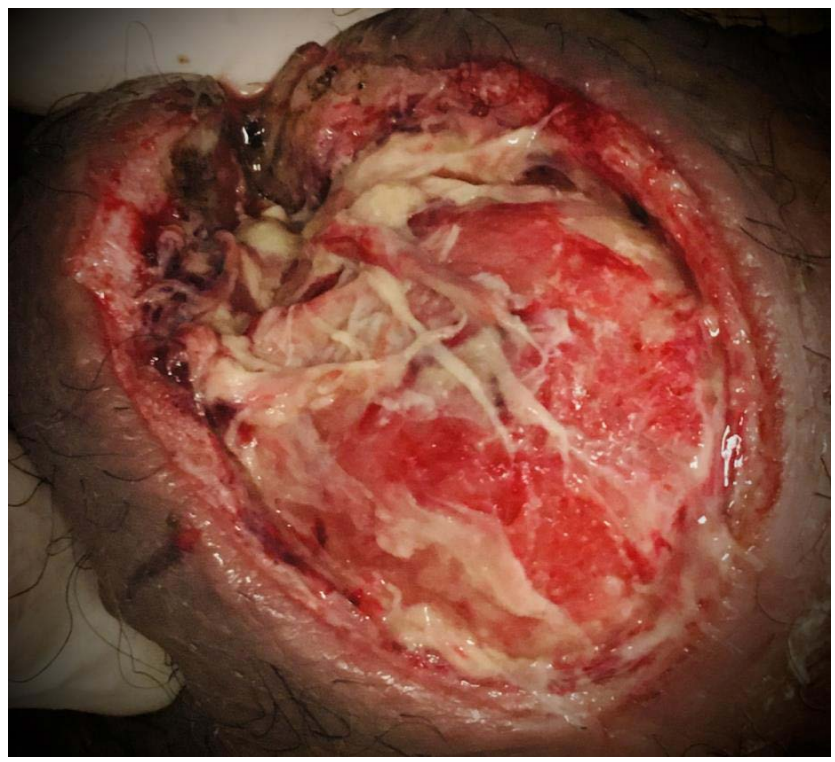

Figure 5. Inflamed and edematous scrotum.

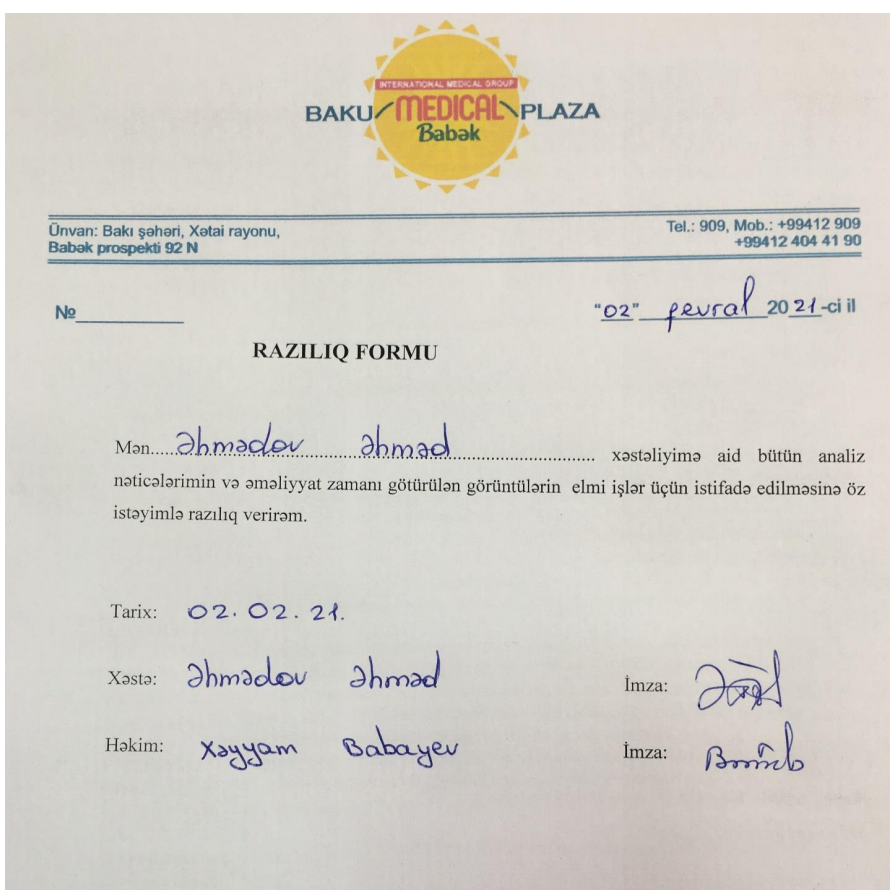

Figure 6. Consent form.

Pathological examination demonstrated no malignancy. A severely inflamed urachus was found.

\section{Discussion}

Diverticulitis is one of the most common bowel emergencies to occur with acute abdomen. Acute diverticulitis constitutes $3.8 \%$ of the causes of abdominal pain in patients admitted to the emergency department [4]. Complicated diverticulitis includes diverticulitis with abscess, obstruction, diffuse peritonitis (free perfora- 
tion), or fistulas between the colon and adjacent structures. Perforations that occur in $1 \%-2 \%$ of patients with acute diverticulitis may lead to local abscess and fistula formation [5]. The rate of fistula formation is around $14 \%$ after an episode of acute diverticulitis [6]. They occur when a diverticular abscess breaches the wall integrity of the adjacent anatomic structure. Fistula from diverticulitis can be seen as, in the order of decreasing frequency, colovesical, colo-enteric and colo-uterine forms [7]. A colo-urachal fistula was seen in our patient.

The urachus is an embryonic tube that connects the upper portion of the bladder to the umbilicus and usually obliterates during embryonic development stages forming the median umbilical ligament. Abnormal obliteration results in many anomalies, including urachal fistula, diverticulum, sinus, and urachal cyst, which is the most common variety [8].

Unless complicated, the urachal cysts are usually small, silent, and asymptomatic. A urachal cyst may have complications such as infection, bleeding within the cyst, intraperitoneal rupture, intestinal fistula, intestinal obstruction, and a high malignant degeneration incidence. [9]. Patients age 55 years or older and hematuria were the strongest predictors of urachal malignancy [10].

In our patient, the cyst was located in the hypogastric region and adhered to the sigmoid colon. The most common presenting symptom in our patient was fever, scrotal and subcutaneous crepitation. Diagnosis is usually made following exploratory laparotomy for unexplained abdominal pain. Infection complications include sepsis, fistula formation, and rupture leading to peritonitis [11]

Ultrasound scan (USS) can help to make a diagnosis in 77\% of patients. [12]. In our case, the USS was not specific, and a CT scan was used to help make the diagnosis.

Due to the risk of carcinoma, the most appropriate treatment is complete removal of the cyst. [13]. Open excision was applied to our patient, and the excised cyst was sent to pathology.

\section{Conclusion}

Although it is rare and its symptoms are non-specific, urachal abscess should be suspected if there are persistent fever and suprapubic pain, especially in patients with diverticula. It is recommended to remove it when the diagnosis is made, considering the complications that may occur.

\section{Conflicts of Interest}

We have no conflict of interest.

\section{Note}

Consent of the patient was obtained (Figure 6).

\section{References}

[1] Rapoport, D., et al. (2007) Urachal-Sigmoid Fistula Associated with Diverticular 
Disease. Canadian Urological Association Journal, 1, 52-54.

https://doi.org/10.5489/cuaj.40

[2] Peters, A.L., et al. (2012) A Colo-Urachal-Cutaneous Fistula in an 88-Year-Old Male. International Journal of Surgery Case Reports, 3, 55-58.

https://doi.org/10.1016/j.ijscr.2011.10.013

[3] Charles Brunicardi, F., et al. (2015) Schwartz's Principles of Surgery. 10th Edition, McGraw-Hill, New York.

[4] Siewert, B. and Raptopoulos, V. (1994) CT of the Acute Abdomen: Findings and Impact on Diagnosis and Treatment. American Journal of Roentgenology, 163, 1317-1324. https://doi.org/10.2214/ajr.163.6.7992721

[5] West, A.B. and Losada, M. (2004) The Pathology of Diverticulosis Coli. Journal of Clinical Gastroenterology, 38, S11-S16. https://doi.org/10.1097/01.mcg.0000124005.07433.69

[6] Sessa, B., et al. (2016) Acute Perforated Diverticulitis: Assessment with Multidetector Computed Tomography. Seminars in Ultrasound, CT and MRI, 37, 37-48. https://doi.org/10.1053/j.sult.2015.10.003

[7] Suros, J. and Lee, R.A. (1973) Pneumoretroperitoneum, Pneumomediastinum, and Subcutaneous Emphysema. Complications of Acute, Perforated Diverticulitis. Minnesota Medicine, 56, 747-749.

[8] Blichert-Toft, M. and Nielsen, O.V. (1971) Diseases of the Urachus Simulating Intra-Abdominal Disorders. The American Journal of Surgery, 122, 123-128. https://doi.org/10.1016/0002-9610(71)90365-5

[9] Yiee, J.H., et al. (2007) A Diagnostic Algorithm for Urachal Anomalies. Journal of Pediatric Urology, 3, 500-504. https://doi.org/10.1016/j.jpurol.2007.07.010

[10] Ashley, R.A., Inman, B.A., Routh, J.C., et al. (2007) Urachal Anomalies: A Longitudinal Study of Urachal Remnants in Children and Adults. The Journal of Urology, 178, 1615-1618. https://doi.org/10.1016/j.juro.2007.03.194

[11] Ekwueme, K.C. and Parr, N.J. (2009) Infected Urachal Cyst in an Adult: A Case Report and Review of the Literature. Cases Journal, 2, Article No. 6422. https://doi.org/10.4076/1757-1626-2-6422

[12] Yoo, K.H., Lee, S.-J. and Chang, S.-G. (2006) Treatment of Infected Urachal Cysts. Yonsei Medical Journal, 47, 423-427. https://doi.org/10.3349/ymj.2006.47.3.423

[13] Johnson, D.E., et al. (1985) Urachal Carcinoma. Urology, 26, 218-221. https://doi.org/10.1016/0090-4295(85)90112-8 